Estimation of the effects of countermeasures on different types of accidents in the presence of regression effects

Stig Danielsson

Reprint from Accident Analysis \& Prevention, Vol 20, No 4, pp. 289-298, 1988 


\title{
ESTIMATIGN OF THE EFFECTS OF COUNTERMEASURES ON DIFFERENT TYPES OF ACCIDENTS IN THE PRESENCE OF REGRESSION EFFECTS
}

\author{
STIG DANIELSSON \\ Swedish Road and Traffic Research Institute (VTI), S-581 01 Linköping, Sweden
}

(Received 23 August 1985; in revised form 5 June 1987)

\begin{abstract}
In an earlier paper [Danielsson, 1986], we studied the problem of estimating the safety effect of a countermeasure on the expected number of accidents at road junctions when highaccident sites are selected for the study. Often, however, the countermeasure leads to varying effectiveness on different types of accidents. This paper is a generalization in that we estimate effects of countermeasures for each type of accident. A major result, for which empirical support is provided, is that the expected regression effect is the same for all types of accidents.
\end{abstract}

\section{INTRODUCTION}

In an earlier paper [Danielsson, 1986], we described the problem of the regression effect which can occur in before-and-after studies [for detailed descriptions see Brüde and Larsson, 1982; Hauer, 1980a, 1980b; Hauer, 1986; and Hauer and Persaud, 1983]. In the paper, we proposed a method of purely estimating the effects of countermeasures despite the presence of regression effects.

Unfortunately, there are in practice further complications with the regression effects. Assume, for example, that we wish to study the number of accidents at road junctions to determine whether a particular countermeasure has any (positive) effect. Naturally, the effectiveness of the countermeasure most often varies between different types of accident. The total effect (on all types of accidents) can then be interpreted as the average effect. One possible way to estimate this effect is obtained if junctions are selected at random and an estimate then made of the total (constant) effect. In many cases, however, only those junctions which are especially prone to accidents are selected, so that it is hardly possible to expect that the accident composition at the selected junctions is "normal." An estimate of the total effect from such a sample may therefore be extremely misleading.

One way of avoiding this complication is to estimate the effect for each type of accident. If this can be done, it is then possible to estimate the total effect for any accident composition at the junctions.

\section{FORMULATION OF THE PROBLEM}

Consider a junction which under normal conditions has mean values $m_{1}, m_{2}, \ldots$, $m_{p}$ per time period for $p$ mutually exclusive types of accident. Examples of such types are (for cars) single-vehicle accidents, rear-end collisions, overtaking accidents, etc. When introducing a countermeasure, we assume that the expected numbers of accidents are changed to

$$
\alpha_{1} m_{1}, \alpha_{2} m_{2}, \ldots, \alpha_{p} m_{p}
$$

The total expected number of accidents

$$
m=m_{1}+m_{2}+\ldots+m_{p}
$$


is then changed to $\alpha m$, where

$$
\alpha=\frac{\alpha_{1} m_{1}+\alpha_{2} m_{2}+\ldots+\alpha_{p} m_{p}}{m}
$$

We call $(1-\alpha)$ the total safety effect of the countermeasure, while $\left(1-\alpha_{1}\right), \ldots$, $\left(1-\alpha_{p}\right)$ are the effects on the different types of accidents.

If a junction is selected at random from a population of similar junctions, a correct estimate of $\alpha$ can be obtained by studying the effect of the countermeasure on the total number of accidents. The estimate of $(1-\alpha)$ can then be regarded as the estimated effect of the countermeasure for an average junction. This is obviously not true if the junction is not selected at random. In such a case, it would be necessary to try to estimate the individual effects $1-\alpha_{1}, 1-\alpha_{2}, \ldots, 1-\alpha_{p}$, and then to use these to estimate the total effect $1-\alpha$ for a given junction.

Consider a period before the implementation of a countermeasure and let

$$
\begin{aligned}
X_{j}= & \text { the number of accidents of type } j \text { occurring at the junction studied, } \\
& j=1,2, \ldots, p \text {. }
\end{aligned}
$$

We make the assumption that different $X_{j}$ are independent and Poisson distributed with parameters $m_{1}, m_{2}, \ldots, m_{p}$ respectively. We also define the total number of accidents

$$
X=\sum_{j=1}^{p} X_{j}
$$

which is, of course, Poisson distributed with the parameter

$$
m=\sum_{j=1}^{p} m_{j}
$$

We further assume that the junction has been selected for study because the total number of accidents is large, i.e. that

$$
X \geq k .
$$

The conditional distribution for the total number of accidents at a selected junction is thus what can be termed truncated Poisson [see Danielsson, 1986; Hauer, 1980b; and Hauer and Persaud, 1983] according to

$$
p_{r}^{\prime}(m)=P(X=r \mid X \geq k)=\frac{p_{r}(m)}{q_{k}(m)} ; \quad r=k, k+1, \ldots
$$

where

$$
p_{r}(m)=e^{-m} \frac{m^{r}}{r !} ; \quad q_{k}(m)=\sum_{r=k}^{\infty} p_{r}(m)
$$

Now we are interested not only in the (conditional) distribution for $X$ but mainly in the distribution for $\left(X_{1}, X_{2}, \ldots, X_{p}\right)$ given that $X \geq k$.

The simultaneous distribution

$$
p^{\prime}\left(r_{1}, r_{2}, \ldots, r_{p}\right)=P\left(X_{1}=r_{1}, X_{2}=r_{2}, \ldots, X_{p}=r_{p} \mid X \geq k\right)
$$


becomes

$$
p^{\prime}\left(r_{1}, \ldots, r_{p}\right)=\frac{p_{r_{1}}\left(m_{1}\right) \cdot p_{r_{2}}\left(m_{2}\right) \ldots p_{r_{p}}\left(m_{p}\right)}{q_{k}(m)} ; \quad \begin{aligned}
\text { all } r_{j} & =0,1,2, \ldots, \\
r_{1} & +r_{2}+\ldots+r_{p} \geq k .
\end{aligned}
$$

\section{ESTIMATION OF EXPECTED REGRESSION EFFECTS AND COUNTERMEASURE EFFECTS FOR DIFFERENT TYPES OF ACCIDENT}

Before studying the estimation procedures in detail, it can be instructive to consider a practical example.

\subsection{A practical example illuminating the regression effects}

In the example in Table 1, we consider rural junctions in Sweden which have been unaltered during the period 1977-1982. We study the total number of accidents but also the accidents involving personal injury. The years 1977-1979 are regarded as the before period, and the selection criterion $(X \geq k)$ is based on the total number of accidents. The years 1980-1982 are regarded as the after period, and by comparing the before and after accidents we have estimates of the regression effects, since no countermeasure has been implemented at the junctions during 1977-1982.

The estimated regression effects in Table 1 are fairly high for different selection criteria. We can also notice that the total accident number is lower in the after period than in the before period. This is probably due to a general trend in the accident development. Therefore, the true regression effects are lower than those calculated in the table, but we have not made any corrections.

The results in Table 1 can be formulated in the following hypothesis: the regression effects are the same for all types of accidents as for the total number of accidents. Since an example is not a proof, this hypothesis is proved in the next section.

\subsection{Expected regression effects for different types of accidents}

In Danielsson [1986], we showed that the expected regression effect for the total number of accidents is

$$
R=\frac{\mu_{k}(m)-m}{\mu_{k}(m)}=\frac{p_{k-1}(m)}{q_{k-1}(m)}
$$

where

$$
\mu_{k}(m)=E[X \mid X \geq k] .
$$

\begin{tabular}{|c|c|c|c|c|c|c|c|}
\hline \multirow[b]{2}{*}{$\begin{array}{c}\text { Junctions During } \\
1977-1979 \\
\text { Which Had the } \\
\text { Total Number } \\
\text { of Accidents }\end{array}$} & \multirow[b]{2}{*}{$\begin{array}{l}\text { Number of } \\
\text { Junctions }\end{array}$} & \multicolumn{2}{|c|}{$\begin{array}{l}\text { Number of } \\
\text { Accidents } \\
1977-1979\end{array}$} & \multicolumn{2}{|c|}{$\begin{array}{l}\text { Number of } \\
\text { Accidents } \\
1980-1982\end{array}$} & \multicolumn{2}{|c|}{$\begin{array}{l}\text { Estimated Regression } \\
\text { Effects for the Number } \\
\text { of Accidents }\end{array}$} \\
\hline & & $\begin{array}{l}\text { Total } \\
\text { (b) }\end{array}$ & $\begin{array}{l}\text { Involving } \\
\text { Personal } \\
\text { Injury }\left(\mathrm{b}^{\prime}\right)\end{array}$ & $\begin{array}{l}\text { Total } \\
\text { (a) }\end{array}$ & $\begin{array}{l}\text { Involving } \\
\text { Personal } \\
\text { Injury }\left(a^{\prime}\right)\end{array}$ & $\begin{array}{c}\text { Total } \\
(b-a) / b\end{array}$ & $\begin{array}{l}\text { Involving } \\
\text { Personal } \\
\text { Injury } \\
\left(\mathrm{b}^{\prime}-\mathrm{a}^{\prime}\right) / \mathrm{b}^{\prime}\end{array}$ \\
\hline$\geq 5$ & 61 & 358 & 137 & 195 & 76 & 0.46 & 0.45 \\
\hline$\geq 4$ & 113 & 566 & 215 & 318 & 123 & 0.44 & 0.43 \\
\hline$\geq 3$ & 232 & 923 & 356 & 504 & 194 & 0.45 & 0.46 \\
\hline$\geq 2$ & 518 & 1495 & 582 & 816 & 314 & 0.45 & 0.46 \\
\hline$\geq 1$ & 1193 & 2170 & 841 & 1313 & 507 & 0.39 & 0.40 \\
\hline$\geq 0$ & 2390 & 2170 & 841 & 1887 & 715 & 0.13 & 0.15 \\
\hline
\end{tabular}

Table 1. Estimated regression effects for the total number of accidents and accidents involving personal injury at rural junctions in Sweden 
We now want to determine the regression effect for accident type $j$, i.e.

$$
R_{j}=\frac{\mu_{k}\left(m_{j}\right)-m_{j}}{\mu_{k}\left(m_{j}\right)}
$$

where

$$
\mu_{k}\left(m_{j}\right)=E\left[X_{j} \mid X \geq k\right] .
$$

Conditional to $X=r$ it holds that $X_{j}$ is binomial distributed with expected value $r \cdot m_{j} / m$. Therefore,

$$
\begin{aligned}
\mu_{k}\left(m_{j}\right) & =E\left[X_{j} \mid X \geq k\right]=\sum_{r=k}^{\infty} p_{r}^{\prime}(m) E\left[X_{j} \mid X \geq k, X=r\right] \\
& =\sum_{r=k}^{\infty} p_{r}^{\prime}(m) E\left[X_{j} \mid X=r\right]=\sum_{r=k}^{\infty} p_{r}^{\prime}(m) \cdot r \frac{m_{j}}{m} \\
& =\frac{m_{j}}{m} E[X \mid X \geq k]=\frac{m_{j}}{m} \cdot m \frac{q_{k-1}(m)}{q_{k}(m)}=m_{j} \frac{q_{k-1}(m)}{q_{k}(m)} .
\end{aligned}
$$

By substituting (7) into (6) we get

$$
R_{j}=1-\frac{q_{k}(m)}{q_{k-1}(m)}=\frac{p_{k-1}(m)}{q_{k-1}(m)}=R .
$$

This means that the expected regression effect is the same for all types of accidents. The result may appear slightly surprising, but with a little afterthought it is natural and even intuitive. The "extra" accidents generated by the condition $X \geq k$ obviously have to be distributed proportionally to $m_{1}, m_{2}, \ldots, m_{p}$ for the different types of accidents.

\subsection{Estimation of the expected accident numbers for a single junction}

In order to estimate the different $m_{j}$ for a single junction, we construct the likelihood function for $x_{1}, x_{2}, \ldots, x_{p}$ (which are the observed number of accidents of different types)

$$
\mathscr{L}=p^{\prime}\left(x_{1}, \ldots, x_{p}\right)
$$

We then seek the $m_{j}$ which maximize [see (4)]

$$
\begin{aligned}
l & =\ln (\mathscr{L})=\sum_{j=1}^{p} \ln \left(p_{x_{j}}\left(m_{j}\right)\right)-\ln \left(q_{k}(m)\right) \\
& =\sum_{j=1}^{p} x_{j} \ln m_{j}-\sum_{j=1}^{p} \ln \left(x_{j} !\right)-\ln \left(\sum_{r=k}^{\infty} \frac{m^{r}}{r !}\right) .
\end{aligned}
$$

By differentiating (9) we obtain

$$
\frac{\partial l}{\partial m_{j}}=\frac{x_{j}}{m_{j}}-\frac{\sum_{r=k}^{\infty} \frac{m^{r-1}}{(r-1) !}}{\sum_{r=k}^{\infty} \frac{m^{r}}{r !}}=\frac{x_{j}}{m_{j}}-\frac{q_{k-1}(m)}{q_{k}(m)} .
$$

If the derivatives in (10) are set equal to 0 we obtain

$$
x_{j}=m_{j} \frac{q_{k-1}(m)}{q_{k}(m)} ; j=1,2, \ldots, p .
$$


The right-hand term in (11) is, of course, the earlier calculated $E\left[X_{j} \mid X \geq k\right]$, i.e. the maximum likelihood (ML) estimates above are identical to the moment estimates.

We have shown earlier [Danielsson, 1986] that the ML estimate for $m$ can be calculated from the equation

$$
x=m \cdot \frac{q_{k-1}(m)}{q_{k}(m)},
$$

and this result is, of course, also obtained if the equations in (11) are summed.

From (11) and (12) we can state that

$$
x_{j}=m_{j} \frac{q_{k-1}(m)}{q_{k}(m)}=m_{j} \cdot \frac{x}{m},
$$

i.e. if $m_{j}^{*}, j=1,2, \ldots, p$, and $m^{*}$ are the ML estimates, we obtain the following simple relations

$$
m_{j}^{*}=m^{*} \cdot \frac{x_{j}}{x}
$$

To obtain the ML estimate of all $m_{j}$, we therefore need only solve one equation numerically [the ML equation for $m$ in (12)]. The simple relations in (14) then give the required estimates.

Since according to (8) the regression effects are the same for all types of accidents, it is sufficient to estimate $R$ according to

$$
R^{*}=\frac{x-m^{*}}{x}
$$

Finally, we can also determine an intuitive estimate of $m_{j}$ by analogy with Hauer's estimate of $m$ [Hauer, 1980a, 1980b]. Hauer has proposed the following estimate of $m$

$$
\hat{m}= \begin{cases}0 & \text { if } x=k \\ x & \text { if } x>k\end{cases}
$$

In agreement with (16) we estimate $m_{j}$ with

$$
\hat{m}_{j}= \begin{cases}0 & \text { if } x=k \\ x_{j} & \text { if } x>k\end{cases}
$$

The estimator $\hat{m}_{j}$ is unbiased since [see (7)]

$$
\begin{aligned}
E\left[\hat{m}_{j}\right] & =\mu_{k}\left(m_{j}\right)-p_{k}^{\prime}(m) k \cdot \frac{m_{j}}{m}=m_{j}\left(\frac{q_{k-1}(m)}{q_{k}(m)}-\frac{k}{m} \frac{p_{k}(m)}{q_{k}(m)}\right) \\
& =m_{j}\left(\frac{q_{k-1}(m)-p_{k-1}(m)}{q_{k}(m)}\right)=m_{j},
\end{aligned}
$$

where

$$
k p_{k}(m)=m p_{k-1}(m)
$$

\subsection{Simultaneous estimates of regression and countermeasure effects}

Assume that at $n$ junctions we wish to introduce a countermeasure which for different types of accidents has the constant effects $1-\alpha_{1}, 1-\alpha_{2}, \ldots, 1-\alpha_{p}$, respectively. 
Regardless of the nature of the site, we assume that the effects are fixed. Of course, in many practical situations this is unrealistic because remedial measures interact with their environment. We apply the following notation for junction no. $i$ :

$X_{j i}=$ the number of accidents of type $j$ before the countermeasure is implemented;

$Y_{j i}=$ the corresponding number of accidents after the countermeasure is implemented (during an equal duration of time);

$X_{\cdot i}=\sum_{j=1}^{p} X_{j i}=$ the total number of accidents in the before period;

$Y_{\cdot i}=\sum_{j=1}^{p} Y_{j i}=$ the total number of accidents in the after period.

In Section 2, we studied the distributions for variables of the type $X_{j i}\left(X_{\cdot i}\right.$ is assumed to be truncated in $k_{i}$ ). We assume that $Y_{j i}$ is Poisson distributed with expected values $\alpha_{j} \cdot m_{j i}$ where

$m_{j i}=$ the expected number of accidents of type $j$ before implementation at the junction studied.

Furthermore, it holds (with assumed independence between the $Y_{j i}$ ) that $Y_{{ } i}$ is Poisson distributed with expected values $\alpha_{\cdot i} \cdot m_{\cdot i}$ where

$$
\begin{aligned}
& m_{\cdot i}=\sum_{j=1}^{p} m_{j i}=\begin{array}{l}
\text { the expected total number of accidents before implementation } \\
\text { at the junction, }
\end{array} \\
& \alpha_{\cdot i}=\sum_{j=1}^{p} \frac{m_{j i}}{m_{\cdot i}} \alpha_{j}=\text { the total effect of the countermeasure at junction no. } i .
\end{aligned}
$$

Now, the problem is to estimate the countermeasure parameters $\alpha_{1}, \ldots, \alpha_{p}$ and $\alpha_{. i}$ by using directly all the data $x_{j i}$ and $y_{j i}$ from all junctions. In the appendix the following maximum likelihood estimates (ML estimates) of $\alpha_{j}$ and $m_{j i}$ are derived

$$
\begin{gathered}
\alpha_{j}=\frac{\sum_{i=1}^{n} y_{j i}}{\sum_{i=1}^{n} m_{j i}} ; j=1,2, \ldots, p \\
m_{j i} \cdot \frac{q_{k_{i}-1}\left(m_{\cdot i}\right)}{q_{k_{i}}\left(m_{\cdot i}\right)}+\alpha_{j} m_{j i}=x_{j i}+y_{j i} ; \quad \begin{array}{l}
j=1,2, \ldots, p \\
i=1,2, \ldots, n .
\end{array}
\end{gathered}
$$

This complicated equation system can be solved by a rather simple numerical method, which is outlined in the appendix.

We observe that the estimator of $\alpha_{j}$ in (19) is of the same type of quotient as in the case where a common effect for all accidents is estimated [see Danielsson, 1986]. The argument in Danielsson [1986] for judging the uncertainty of $\alpha_{j}^{*}$ will be fully equivalent also here. We will therefore not concern ourselves with these details.

Finally, we note that it is also possible to estimate $\alpha_{j}$ by using the intuitive estimates of $m_{j i}$. The reasonable estimate of $\alpha_{j}$ is of course

$$
\hat{\alpha}_{j}=\frac{\sum_{i=1}^{n} y_{j i}}{\sum_{i=1}^{n} \hat{m}_{j i}}
$$


where

$$
\hat{m}_{j i}= \begin{cases}0 & \text { if } x_{\cdot i}=k_{i} \\ x_{j i} & \text { if } x_{\cdot i}>k_{i}\end{cases}
$$

\section{COMMENTS ABOUT THE PRACTICAL APPLICABILITY OF THE ESTIMATION METHODS}

In the preceding section we have derived the ML estimators of the different $\alpha_{j}$. The estimators are rather complicated to handle and there is a need for simpler and quicker estimation methods even though they are less efficient. We begin the discussion with the ML estimators and then successively introduce some simplifications.

(i) ML estimators. The estimator of $\alpha_{j}$ derived from (19) and (20) is rather complicated, even if the multidimensional problem can be reduced to a sequence of onedimensional problems as described in the appendix.

The form of the ML estimator (19) is simple provided the estimators of $m_{j i}$ are determined. Thus, simplified estimators can be obtained by replacing the denominator in (19) with estimators of $m_{j i}$ other than the ML estimators.

(ii) ML-like estimators. In (21) we have proposed a simple estimator of $\alpha_{j}$ which does not require any complex calculation. The estimator of $\alpha_{j}$ can be formulated

$\hat{\alpha}_{j}=$ the total number of type $j$ accidents in the after period divided by the corresponding total number in the before period for the sites which fulfill the selection criteria plus one type $j$ accident.

Another estimator can be derived from (14). Using only the before data for estimating $m_{j i}$, the ML estimator is

$$
m_{j i}^{*}=m_{\cdot i}^{*} \frac{x_{j i}}{x_{\cdot i}}
$$

where $m_{\cdot i}^{*}$ easily can be calculated from [see (12)]

$$
x_{\cdot i}=m_{\cdot i} \frac{q_{k_{i}-1}\left(m_{\cdot i}\right)}{q_{k_{i}}\left(m_{\cdot i}\right)} .
$$

Then, the estimator of $\alpha_{j}$ is

$$
\alpha_{j}^{*}=\frac{\sum_{i=1}^{n} y_{j i}}{\sum_{i=1}^{n} m_{j i}^{*}}
$$

(iii) Estimators by explicit use of the regression effect. If the regression effect $R_{i}$ in (5) for the total number of accidents at each site is known, then $\alpha_{j}$ can be estimated from

$$
\tilde{\alpha}_{j}=\frac{\sum_{i=1}^{n} y_{j i}}{\sum_{i=1}^{n}\left(1-R_{i}\right) x_{j i}} .
$$

The result follows from the definitions (5) and (6) and from the fact that all accident types have the same regression effect [see (8)]. 
In practice, the different $R_{i}$ very seldom are known. However, in some cases it is possible to assess the regression effect and the estimate in (25) can be determined with this estimate of $R_{i}$. One way to estimate $R_{i}$ is to use the "Strike method" proposed by Brüde and Larsson [1982]. Formula (25) is especially simple when all sites have the same regression effect $R$. Then we have

$$
\tilde{\alpha}_{j}=\frac{\sum_{i=1}^{n} y_{j i}}{(1-R) \sum_{i=1}^{n} x_{j i}} .
$$

(iv) An illustrative example. From the material in Table 1, 10 junctions are selected, all with total accident numbers of at least 5 in the before period. The selected junctions are presented in Table $2 \mathrm{a}$.

Let $1-\alpha_{1}, 1-\alpha_{2}$, and $1-\alpha$ be the countermeasure effects for accidents involving personal injury, accidents not involving personal injury, and all the accidents respectively. It is interesting to compare the estimates $\hat{\alpha}_{j}, \alpha_{j}^{*}$ and $\tilde{\alpha}_{j}$ proposed in (21), (24), and (26). It is worth noting that there is no real countermeasure effect, but instead we seek to estimate a general downward effect in the whole population of about $15 \%$.

It is very easy to compute the estimates proposed in (21). Consider e.g. the accidents involving personal injury. Then

$$
\hat{\alpha}_{1}=\frac{14}{24-5}=0.74
$$

To compute $\alpha_{1}^{*}$ in (24) it is necessary to solve the equation

$$
10=m_{\cdot 1}^{*} \frac{q_{4}\left(m_{\cdot 1}^{*}\right)}{q_{5}\left(m_{\cdot 1}^{*}\right)}
$$

for junction No. 1, and then the corresponding equations for the other junctions. The definition of $q_{k}$ can be found in (2). The solution of the equation above is

$$
m_{\cdot 1}^{*}=9.8
$$

\begin{tabular}{|c|c|c|c|c|c|c|}
\hline \multirow[b]{2}{*}{ Junction No. } & \multicolumn{3}{|c|}{ Number of Accidents 1977-1979 } & \multicolumn{3}{|c|}{ Number of Accidents $1980-1982$} \\
\hline & Total & $\begin{array}{l}\text { Involving } \\
\text { Personal } \\
\text { Injury }\end{array}$ & $\begin{array}{l}\text { Not Involving } \\
\text { Personal } \\
\text { Injury }\end{array}$ & Total & $\begin{array}{c}\text { Involving } \\
\text { Personal } \\
\text { Injury }\end{array}$ & $\begin{array}{c}\text { Not Involving } \\
\text { Personal } \\
\text { Injury }\end{array}$ \\
\hline 1 & 10 & 4 & 6 & 3 & 1 & 2 \\
\hline 2 & 8 & 5 & 3 & 8 & 5 & 3 \\
\hline 3 & 7 & 3 & 4 & 3 & 2 & 1 \\
\hline 4 & 7 & 2 & 5 & 3 & 0 & 3 \\
\hline 5 & 6 & 2 & 4 & 2 & 1 & 1 \\
\hline 6 & 6 & 3 & 3 & 5 & 2 & 3 \\
\hline 7 & 5 & 1 & 4 & 3 & 1 & 2 \\
\hline 8 & 5 & 1 & 4 & 4 & 1 & 3 \\
\hline 9 & 5 & 1 & 4 & 3 & 1 & 2 \\
\hline 10 & 5 & 2 & 3 & 2 & 0 & 2 \\
\hline All junctions & 64 & 24 & 40 & 36 & 14 & 22 \\
\hline
\end{tabular}

and then

$$
m_{11}^{*}=9.8 \cdot \frac{4}{10}=3.92
$$

Table 2a. Number of accidents for selected junctions divided with respect to personal injury 


\begin{tabular}{lccc}
\hline & \multicolumn{3}{c}{$\begin{array}{c}\text { Estimated Countermeasure Effect for the } \\
\text { Number of Accidents }\end{array}$} \\
\cline { 2 - 4 } & Total & $\begin{array}{c}\text { Involving } \\
\text { Personal Injury }\end{array}$ & $\begin{array}{c}\text { Not Involving } \\
\text { Personal Injury }\end{array}$ \\
\hline No correction method $\left(\alpha^{\prime}\right)$ & 0.44 & 0.42 & 0.45 \\
Hauer type of correction $(\hat{\alpha})$ & 0.18 & 0.26 & 0.12 \\
ML-type of correction $\left(\alpha^{*}\right)$ & 0.02 & 0.12 & -0.06 \\
"Known" regression effect $(\tilde{\alpha})$ & 0.13 & 0.10 & 0.15 \\
\hline
\end{tabular}

For the observations $8,7,6$, and 5 the corresponding $m$. -values are $7.4,6.0,3.8$, and 0 . Then it is easy to compute all $m_{1 i}^{*}$ and we have $\Sigma m_{1 i}^{*}=16.0$. Therefore

$$
\alpha_{1}^{*}=\frac{14}{16.0}=0.88
$$

From Table 1, we can conclude that the regression effect is about $35 \%$ (since there is general downward secular trend of about 15\%). Therefore we have from (26) (assuming the same regression effect at all sites)

$$
\tilde{\alpha}_{1}=\frac{14}{0.65 \cdot 24}=0.90 \text {. }
$$

Without any compensation of the regression effect, the estimate of $\alpha_{1}$ is

$$
\alpha_{1}^{\prime}=\frac{14}{24}=0.58
$$

The results of all computations are presented in Table $2 \mathrm{~b}$. It is obvious that the countermeasure effects (in this case, the secular changes) are seriously overestimated if no correction method is applied. The various correction methods give similar results, but the estimates are rather unstable. An exception is the estimate $\tilde{\alpha}$, which, since it utilizes the "known" regression effect, is quite reasonable.

\section{REFERENCES}

Brüde U. and Larsson J., The regression-to-mean effect. Some empirical examples concerning accidents at road junctions. VTI Report 240, 1982.

Danielsson S., A comparison of two methods for estimating the effect of a countermeasure in the presence of regression effects. Accid. Anal. Prev. 18, 13-23, 1986.

Hauer E., Bias by selection: Overestimation of the effectiveness of safety countermeasures caused by the process of selection for treatment. Accid. Anal. Prev. 12, 113-117, 1980a.

Hauer E., Selection for treatment as a source of bias in before-and-after studies. Traffic Eng. Control 8/9, 419-421, 1980b.

Hauer E., On the estimation of the expected number of accidents. Accid. Anal. Prev. 18, 1-12, 1986.

Hauer E. and Persaud B., Common bias in before-and-after accident comparisons and its elimination. Transpn. Res. Rec. 905, 164-174, 1983.

\section{APPENDIX}

Maximum likelihood estimates of $\alpha_{j}$ and $m_{j i}$ in Section 3.4

We wish to estimate the countermeasure parameters $\alpha_{1}, \ldots, \alpha_{p}$ and $\alpha_{. i}$ by directly using data from all $n$ junctions. The total likelihood for the observed values $x_{i i}$ and $y_{j i}(j=1,2, \ldots, p, i=1,2, \ldots, n)$ will 
be [see (2) and (4)]

$$
\begin{aligned}
\mathscr{L} & =\prod_{i=1}^{n} p^{\prime}\left(x_{1 i}, x_{2 i}, \ldots, x_{p i}\right) \cdot p_{y_{1 i}}\left(\alpha_{1} m_{1 i}\right) \cdot \ldots \cdot p_{y_{p i}}\left(\alpha_{p} m_{p i}\right) \\
l & =\ln (\mathscr{L})=\sum_{i=1}^{n}\left\{\sum_{j=1}^{p} \ln \left(p_{x_{j i}}\left(m_{j i}\right)\right)-\ln \left(q_{k_{i}}\left(m_{\cdot i}\right)\right)+\sum_{j=1}^{p} \ln \left(p_{y_{j i}}\left(\alpha_{j} m_{j i}\right)\right)\right\} \\
& =\sum_{i=1}^{n}\left\{\sum_{j=1}^{p} x_{j i} \ln m_{j i}-\sum_{j=1}^{p} \ln \left(x_{j i} !\right)-\ln \left(\sum_{r=k i}^{\infty} \frac{\left(m_{\cdot i}\right)^{r}}{r !}\right)\right. \\
& \left.-\sum_{j=1}^{p} \alpha_{j} m_{j i}+\sum_{j=1}^{p} y_{j i} \ln \left(\alpha_{i} \cdot m_{j i}\right)-\sum_{j=1}^{p} \ln \left(y_{j i} !\right)\right\} .
\end{aligned}
$$

Differentiating (A1) gives

$$
\left\{\begin{array}{l}
\frac{\partial l}{\partial \alpha_{j}}=-\sum_{i=1}^{n} m_{j i}+\sum_{i=1}^{n} \frac{y_{j i}}{\alpha_{j}} ; j=1,2, \ldots, p \\
\frac{\partial l}{\partial_{m_{j i}}}=\frac{x_{j i}}{m_{j i}}-\frac{q_{k_{i}-1}\left(m_{\cdot i}\right)}{q_{k_{i}}\left(m_{\cdot i}\right)}-\alpha_{j}+\frac{y_{j i}}{m_{j i}} ; \begin{array}{l}
j=1,2, \ldots, p \\
i=1,2, \ldots, n .
\end{array}
\end{array}\right.
$$

If the derivatives in (A2) are set equal to 0 we obtain

$$
\begin{gathered}
\alpha_{j}=\frac{\sum_{i=1}^{n} y_{j i}}{\sum_{i=1}^{n} m_{j i}} ; j=1,2, \ldots, p, \\
m_{j i} \cdot \frac{q_{k_{i}-1}\left(m_{\cdot i}\right)}{q_{k_{i}}\left(m_{\cdot i}\right)}+\alpha_{j} m_{j i}=x_{j i}+y_{j i} ; \begin{array}{l}
j=1,2, \ldots, p \\
i=1,2, \ldots, n .
\end{array}
\end{gathered}
$$

Summing (A4) with regard to $j$, we obtain

$$
m_{\cdot i} \cdot \frac{q_{k_{i}-1}\left(m_{\cdot \cdot}\right)}{q_{k_{i}}\left(m_{\cdot i}\right)}+\sum_{j=1}^{p} \alpha_{j} m_{j i}=x_{\cdot i}+y_{\cdot i} ; \quad i=1,2, \ldots, n .
$$

Since from definition

$$
\sum_{j=1}^{p} \alpha_{j} m_{j i}=\alpha_{\cdot i} \cdot m_{\cdot i}
$$

where $\alpha_{._{i}}$ is the total effect for junction no. $i$, for each given $\alpha_{{ }_{i}}$ it is now easy to solve $m_{\cdot i}$ from (A5) by using the method proposed by Danielsson [1986]. If we know $\alpha_{j}$ we can then solve $m_{j i}$ from

$$
m_{j i}=m_{\cdot i} \cdot \frac{x_{j i}+y_{j i}}{x_{\cdot i}+y_{\cdot i}-\alpha_{\cdot i} m_{\cdot i}+\alpha_{j} m_{\cdot i}} ; \quad i=1,2, \ldots, n,
$$

which are obtained from (A4), (A5), and (A6).

By assigning different $\alpha_{j}$ and solving the corresponding $m_{j i}$ from (A7) we can test for a solution $m_{j i}$ which must, of course, satisfy (A3).

These $p$ solutions must then satisfy the requirements in (A6) if $m_{j i}$ is to be the ML estimate. If the requirements are not satisfied, new $\alpha_{._{i}}(i=1,1, \ldots, n)$ are tested until the optimal solution is obtained.

Thus, it is possible to solve the complicated equation system in (A3) and (A4) by rather simple methods. Numerical solutions are necessary only for calculating all the $m_{i}$, and in Danielsson [1986] we have presented a method for these calculations. 
